\title{
Suppression of VEGF expression through interruption of the HIF-1 $\alpha$ and Akt signaling cascade modulates the anti-angiogenic activity of DAPK in ovarian carcinoma cells
}

\author{
SUNG TAEK PARK ${ }^{1 *}$, BOH-RAM KIM ${ }^{2 *}$, SUNG HO PARK ${ }^{1}$, JEONG HEON LEE $^{3}$, \\ EUN-JU LEE ${ }^{4}$, SEUNG-HOON LEE ${ }^{5}$ and SEUNG BAE RHO ${ }^{2}$ \\ ${ }^{1}$ Department of Obstetrics and Gynecology, Hallym University, Seoul 150-950; ${ }^{2}$ Research Institute, \\ National Cancer Center, Goyang-si, Gyeonggi-do 410-769; ${ }^{3}$ Department of Obstetrics and Gynecology, \\ Chonbuk National University Medical School, Jeonju 561-712; ${ }^{4}$ Department of Obstetrics and Gynecology, \\ Chung-Ang University School of Medicine/Chung-Ang University Hospital, Seoul 156-755; ${ }^{5}$ Department of \\ Life Science, Yong In University, Yongin-si, Gyeonggi-do 449-714, Republic of Korea
}

Received November 1, 2013; Accepted December 2, 2013

DOI: $10.3892 /$ or.2013.2928

\begin{abstract}
Death-associated protein kinase (DAPK) plays an important role in apoptosis regulation and has been shown to maintain antitumor and metastasis suppressor properties. In the present study, we investigated whether DAPK overexpression may mediate vascular endothelial growth factor (VEGF)/hypoxia-inducible factor-1 $\alpha$ (HIF-1 $\alpha$ ) expression and angiogenic activity in the human carcinoma cell model system. VEGF plays a pivotal role in tumor angiogenesis and tumorigenesis. We found that DAPK significantly downregulated VEGF-induced endothelial cell proliferation, migration and tube formation as well as VEGF receptor-2 (VEGFR-2) phosphorylation in vitro. In addition, DAPK exhibited potent anti-angiogenic activity and clearly decreased the levels of VEGF and HIF-1 $\alpha$ expression, a key regulator for angiogenesis. Notably, our results strongly indicated that DAPK can disturb VEGFR-2 transcriptional activity by inhibiting VEGFR-2 phosphorylation through the PI3K/Akt signaling
\end{abstract}

Correspondence to: Dr Seung Bae Rho, Research Institute, National Cancer Center, 323 Ilsan-ro, Ilsandong-gu, Goyang-si, Gyeonggi-do 410-769, Republic of Korea

E-mail: sbrho@ncc.re.kr

${ }^{*}$ Contributed equally

Abbreviations: DAPK; death-associated protein kinase; VEGF, vascular endothelial growth factor; HIF-1 $\alpha$, hypoxia-inducible factor-1 $\alpha$; PDK1, phosphoinositide-dependent protein kinase-1; mTOR, mammalian target of rapamycin; TSC-2, tuberous sclerosis complex-2; 4E-BP1, eukaryotic translation initiation factor $4 \mathrm{E}$ binding protein 1; HUVECs, human umbilical vein endothelial cells

Key words: death-associated protein kinase, hypoxia-inducible factor-1 $\alpha$ expression, anti-angiogenic activity, vascular endothelial growth factor receptor 2, protein-protein interaction cascade. Collectively, our study identified a novel function of DAPK in regulating cellular VEGF/HIF-1 $\alpha$ activity during tumorigenesis, which may act together with its anti-angiogenic function to inhibit tumor progression.

\section{Introduction}

Death-associated protein kinase (DAPK), a 160-kDa calcium/calmodulin-dependent Ser/Thr kinase protein, is an essential mediator for apoptotic cell death induced by various stimuli, including interferon- $\gamma$ (IFN- $\gamma$ ), tumor necrosis factor- $\alpha$ (TNF- $\alpha$ ), transforming growth factor- $\beta$ (TGF- $\beta$ ) and Fas ligand (1-3). DAPK is a well-known pro-apoptotic protein as well as a tumor suppressor and is involved in a wide variety of cellular apoptotic signaling cascades (3-7). It is also triggered by cell death-regulator genes and deregulated in various tumor types, including head, neck, non-small cell lung and pancreatic cancer (8-10). In addition, the downregulation of DAPK expression is observed in various types of tumors, including B-cell lymphoma $(11,12)$. The death domain of DAPK controls its interactions with tumor necrosis factor receptor-1 (1), FADD (13), RSK (14), the mitogen-activated protein kinase extracellular signal-regulated kinase (ERK), Src (15) and the UNC5H2-dependent receptor (16). Of note, clinical studies have significantly related the loss of DAPK expression with a more malignant type of cancer, while upregulating the metastatic ability of human cancer. In non-small cell lung tumors, DAPK promoter methylation was closely associated with aggressive disease types and poor survival (9,17-19). However, how the cellular activities of DAPK are regulated in vitro remains poorly understood.

The proliferation, migration, blood vessel invasion and expansion of the primary capillary-like tubular network of the endothelial cells during embryogenesis, angiogenesis and vasculogenesis are mostly operated by the vascular endothelial growth factor (VEGF) signaling cascade via the VEGF receptor-2 (VEGFR-2) (20). In tumor angiogenesis and pathogenesis, the tumor microenvironment is one of the most 
important factors for development, in which the environment is often hypoxic. Tumor hypoxia is a condition of oxygen deprivation in carcinoma cells, where hypoxic tumor cells are generally resistant to chemo- and radio-therapy (21). The hypoxic environment leads to genetic instability, which is beneficially related to tumor progression. In addition, hypoxia promotes of hypoxia-inducible factor- $1 \alpha$ (HIF-1 $\alpha)$ protein expression, which stimulates angiogenesis and is correlated with poorer prognosis with the activation of several genes such as VEGF, erythropoietin (EPO) and nitric oxide synthases that are related to metastasis (22). The upregulation of VEGF occurs in most aggressive solid cancers, including ovarian, breast, lung, colon and uterus tumors, and is also closely connected to cancer progression and poor prognosis (23-26). In addition, VEGF and VEGFR-2 overexpression is also closely related to a reduced disease-free survival rate (26) and overall survival with ovarian tumors $(27,28)$. Thus, the precise molecular mechanism for ovarian carcinoma cells remains to be fully elucidated.

For the objective of the present study, we analyzed ovarian carcinoma cells SKOV-3 as a model system in order to examine the functional mechanism of the anti-angiogenic properties of DAPK. We observed that DAPK markedly suppressed the expression of VEGF and HIF-1 $\alpha$ in carcinoma cells. Thus, we investigated a possible molecular mechanism by which DAPK reduced VEGF production in an in vitro model system.

\section{Materials and methods}

Cell lines, culture, chemicals and antibodies. Human epithelial ovarian cancer cell lines SKOV-3 were grown in Dulbecco's modified Eagle's medium (DMEM) supplemented with $10 \%$ fetal bovine serum (FBS). Primary human umbilical vein endothelial cells (HUVECs) were cultured on $0.3 \%$ gelatin-coated dishes (Sigma, St. Louis, MO, USA) using EGM-2 BulletKit medium (Clonetics). Two cell lines were purchased from the American Type Culture Collection (ATCC, Manassas, VA, USA). All cells were cultured under $5 \% \mathrm{CO}_{2}$ at $37^{\circ} \mathrm{C}$. Rapamycin was obtained from Cell Signaling Technology, Inc. (Beverly, MA, USA) and wortmannin and LY294002 were from Sigma. The primary antibodies used in the present study were: anti-DAPK, anti-phospho-specific PI3K, anti-PI3K, anti-phospho-specific Akt, anti-Akt, anti-HIF-1 $\alpha$, anti-phospho-specific PDK-1, anti-PDK-1, antiphospho-specific TSC-2, anti-TSC-2, anti-VEGFR-2 (Santa Cruz Biotechnology, Inc., Santa Cruz, CA, USA), anti-VEGF 121 (Ab-1; Oncogene, Cambridge, MA, USA), anti-phosphospecific mTOR, anti-mTOR, anti-phospho-specific 4E-BP1, anti-4E-BP1, anti-phospho-specific p70S6K, anti-p70S6K (Cell Signaling Technology, Inc.) and anti- $\beta$-actin (Sigma).

$\left[{ }^{3} H\right]$ thymidine incorporation assay. Cell proliferation was calculated by the incorporation of radio-labeled thymidine in trichloroacetic acid as previously described $(29,30)$. HUVECs were plated in 96-well culture plates at densities of $1.5 \times 10^{4}$ cells/well and were labeled with $\left[\right.$ methyl $\left.{ }^{3} \mathrm{H}\right]$ thymidine for the last $4 \mathrm{~h}$ at $0.5 \mu \mathrm{Ci} / \mathrm{ml}$. The cells were filtered by absorption onto paper and were rinsed twice with 5\% TCA and twice with $95 \%$ ethanol. The zone of dried paper corresponding to each well was eliminated with a round hole-punch, and $\left[{ }^{3} \mathrm{H}\right]$ thymidine incorporation was estimated by the liquid scintillation counter (Beckman Instruments).

Cell invasion assay. Transwell cell chambers $(8.0-\mu \mathrm{m}$ pore size; Costar), with 24 wells, were used to assay the cell invasion $(29,31)$. For the invasion assay, the lower surface of a filter was coated with $10 \mu \mathrm{g} / \mathrm{ml}$ of gelatin overnight. M199 containing $1 \%$ FBS with VEGF $(10 \mathrm{ng} / \mathrm{ml})$ was placed in the lower wells. The cells were collected by trypsinization, washed, and re-suspended to $1 \times 10^{5}$ cells in $100 \mu \mathrm{l}$ of fresh DMEM. Subsequently, the cells were inoculated into the upper chamber and incubated for $6 \mathrm{~h}$ at $37^{\circ} \mathrm{C}$. The chamber was fixed with methanol and stained with $10 \mathrm{mg} / \mathrm{ml} \mathrm{H \& E}$. The level of cell invasion was then quantified by calculating the stained cells in five random areas per membrane.

Capillary tube formation assay. The tube formation assay was carried out as previously described using growth factorreduced Matrigel $(30,32)$. In brief, HUVECs $\left(3.5 \times 10^{5}\right)$ were cultured on the surface of the Matrigel. Seeded cells were then incubated with or without $10 \mathrm{ng} / \mathrm{ml}$ of VEGF for $48 \mathrm{~h}$ in M199 containing $1 \%$ FBS. After rinsing, images were captured at $\mathrm{x} 40$ magnification. Tube formation lengths were estimated using an inverted microscope equipped with a digital CCD camera (Zeiss), and quantification was measured using Image Lab imaging software (MCM Design). The experiments were performed in triplicate.

Western blotting. After transfections, cultured cells were collected, rinsed twice with ice-cold PBS and lysed by adding ice-cold RIPA lysis buffer containing a protease inhibitor cocktail (Sigma) at $4^{\circ} \mathrm{C}$ for $1 \mathrm{~h}$. The extract was then transferred to a micro-tube and centrifuged at 15,000 x $\mathrm{g}$ for $10 \mathrm{~min}$. Subsequently, equal amounts of protein were separated with $8-12 \%$ SDS-PAGE, and transferred onto a Hybond-ECL nitrocellulose membrane (GE Healthcare, Little Chalfont, Buckinghamshire, UK). After blocking, the membranes were incubated with the indicated specific primary antibodies at $4^{\circ} \mathrm{C}$ overnight. The membranes were rinsed thrice with TBST buffer and incubated in either goat anti-rabbit or anti-mouse secondary antibodies. Protein bands were developed using an ECL detection system (GE Healthcare).

PI3K activity analysis. PI3 kinase activity was carried out as previously reported $(33,34)$. In brief, cells were grown at a density of $2.6 \times 10^{6}$ cells. Following overnight incubation, cells were transfected according to various concentrations of the indicated specific expression plasmid. The cells were then lysed with ice-cold buffer containing a protease inhibitor cocktail. The lysates were centrifuged at 20,000 x g for $15 \mathrm{~min}$ at $4^{\circ} \mathrm{C}$, and the supernatants were used as the cell lysate. In order to immunoprecipitate PI $3 \mathrm{~K}$, total proteins were incubated with anti-p85 antibody, followed by incubation with protein A-agarose beads for an additional $1 \mathrm{~h}$ at $4^{\circ} \mathrm{C}$. Immunoprecipitates were mixed with a kinase reaction buffer containing $200 \mu \mathrm{g} / \mathrm{ml}$ of phosphatidylinositol and $2 \mu \mathrm{Ci}$ of $\left[{ }^{32} \mathrm{P}\right]$ ATP/assay mixture at $37^{\circ} \mathrm{C}$ for $15 \mathrm{~min}$. The reaction materials were detected using auto-radiography and the radioactive compounds were evaluated using a liquid scintillation counter. 
Yeast two-hybrid system. cDNA, which encodes full-length human DAPK, was introduced into the EcoRI and XhoI restriction enzyme sites of a pGilda-LexA expression shuttle vector. A human full-length VEGFR-1 and VEGFR-2 cDNA was subcloned into the pJG4-5 activation vector in order to generate B42 fusion proteins in the EcoRI and XhoI sites, respectively. Positive interactions were attested by the formation of black colonies on the X-gal-containing medium as previously described (35). The binding activity of the interaction was compared by estimating the relative expression level of $o$-nitrophenyl $\beta{ }_{-}{ }_{D}$-galactopyranoside (ONPG) $\beta$-galactosidase $(35,36)$.

Luciferase reporter-gene assay. In vitro VEGFR-2 promoter activity was carried out as previously reported (36). Briefly, cells at $85 \%$ confluency were transfected with a VEGFR-2 reporter expression plasmid. After lysis with RIPA buffer, total cell lysates were cleared with centrifugation at $14,000 \mathrm{rpm}$ for $15 \mathrm{~min}$ and cell extracts were incubated with the luciferase substrate reagent at room temperature for $30 \mathrm{~min}$ according to the manufacturer's protocols. Then, a $5 \mu \mathrm{l}$ aliquot of each sample was quantitated using a MicroLumat Plus LB96V luminometer.

Statistical analysis. Statistical analysis of the results was performed using the Student's t-test when compared between two groups. Data are presented as the means \pm SD with the error of the mean given for triplicate experiments. The index for statistical significance was $\mathrm{P}<0.05$. The values with $95 \%$ confidence $(\mathrm{P}<0.05)$ are depicted with an asterisk $\left(^{*}\right)$ on each graph. Each experiment was repeated three times with similar results.

\section{Results}

Suppressor DAPK protein inhibits VEGF-induced endothelial cell proliferation, migration and tube formation ability. VEGF, as a potent multifunctional cytokine, is a crucial regulator of the pathological and physiological angiogenesis in tumors. The upregulation of VEGF appears in most solid tumors involved in ovarian, breast, lung, colon and aggressive uterus tumors, and is very closely related to tumor progression and poor prognosis (24-26). Therefore, the suppression of VEGF expression has been shown to suppress tumor growth as well as migration, invasion and metastasis. As a result of these observations, it may be stated that VEGF enhances cell proliferation and migration via multiple signaling pathways. To investigate whether DAPK may inhibit angiogenesis, we first evaluated the effect of DAPK on endothelial cell proliferation by a $\left[{ }^{3} \mathrm{H}\right]$ thymidine incorporation assay. Generally, VEGF-stimulated DNA synthesis was calculated with both untransfected cells and empty vector control-transfected cells and then compared with the uninduced cells. DAPK significantly decreased cell proliferation to $55-65 \%$ that of the expression vector only (control) in these cells. In contrast, the loss of function of DAPK by siRNA-mediated knockdown did not suppress cell proliferation (Fig. 1A).

To address the potential function for DAPK upregulation in decreasing cell proliferation and VEGF-modulated angiogenesis, we additionally observed the invasive cell levels of
DAPK in HUVECs by the Boyden chamber Transwell assay. Cell invasion is also an important step required for angiogenesis. As presented in Fig. 1B, DAPK-siRNA did not inhibit cell invasion of HUVECs, but DAPK markedly suppressed VEGFinduced cell invasion without killing the cells. These data indicate that DAPK may suppress the invasion of endothelial cells by carcinoma cells. In the latter process, endothelial cell capillary-like structures are an important event to become elongated into tubes to form new blood vessels for angiogenesis. We then examined the anti-angiogenic activities of DAPK on VEGF-induced tube formation using an in vitro angiogenesis model system. In the presence of DAPK-siRNA, endothelial cells were almost none affected. In the presence of DAPK, the linear structures of the capillary networks were disrupted (Fig. 1C). Collectively, these results suggest that DAPK suppressed HUVEC invasion and network formation may occur possibly via interruption of VEGF-mediated signaling pathways.

DAPK downregulates PI3K and Akt phosphorylation in a dose-dependent manner. The phosphorylation of PI3K/Akt is an important process for signaling cascade in tumor angiogenesis. Akt plays a pivotal role as a downstream regulator of PI3K and is also regulated by various growth factors, such as epidermal growth factor (EGF), insulin-like growth factor-1 (IGF-1) and transforming growth factor- $\beta 1$ (TGF- $\beta 1$ ). Therefore, we assessed whether DAPK suppressed PI3K and Akt phosphorylation in carcinoma cells. Total cell lysates from the control and transfected cells of various concentrations (0.2-1.0 $\mu \mathrm{g})$ were subjected to immunoblot analysis, which showed that the VEGF-stimulated phosphorylation of PI3K and Akt played a pivotal role in VEGF-induced angiogenesis. As presented in Fig. 2A, the activity of PI3K transfected with various concentrations of DAPK was suppressed in a concentration-dependent manner, with the maximum effect at $1.0 \mu \mathrm{g}$. Following transfection with 0.4-0.6 $\mu \mathrm{g}$ of DAPK, cells showed significantly suppressed phosphorylation of PI3K. We also evaluated the effect of DAPK on the inhibition of Akt, which is one of the major downstream components of PI3K. As expected, DAPK gradually decreased the phosphorylation of Akt in a dose-dependent manner (Fig. 2B). This substrate was similarly able to suppress phosphorylation in HUVECs (data not shown). Taken together, our results strongly suggest that the PI3K/Akt-dependent signal cascades are critically contained in the biological function of DAPK-regulated endothelial cell. This inhibitory effect was comparable to that of some well-known PI3K inhibitors, such as wortmannin and rapamycin, which are well-known for targeting the mammalian target of rapamycin (mTOR) signaling pathways. As indicated in Fig. 2C, DAPK markedly reduced the phosphorylation of Akt (Ser473) and the phosphorylation of eukaryotic translation initiation factor 4E-binding protein 1 (4E-BP1) (Tyr37/46), as well as the phosphorylation of phosphoinositide-dependent protein kinase-1 (PDK1) (Ser241) (Fig. 3C), one of the best characterized targets of the mTOR complex. Subsequently, when DAPK was co-treated with rapamycin, an inhibitor of mTOR, DAPK significantly displayed the effects of deactivation in $\mathrm{p}-4 \mathrm{E}-\mathrm{BP} 1$. These results provide evidence for our former theory that DAPK is a powerful inhibitor of PI3K/Akt activity. 
A

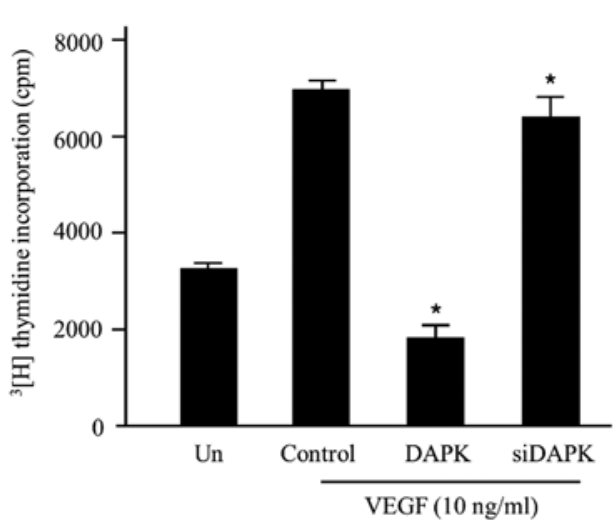

$\mathbf{C}$

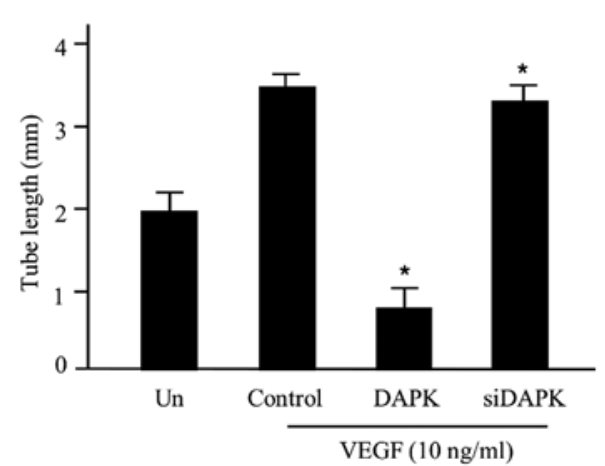

B

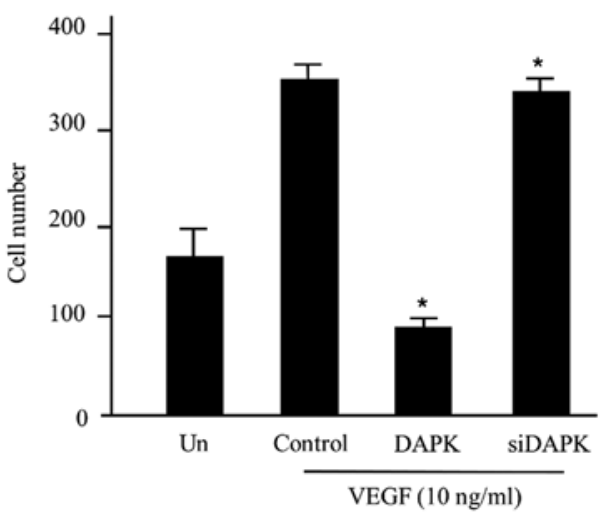

Untreated

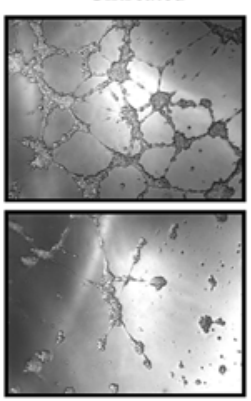

VEGF/DAPK
VEGF/Control
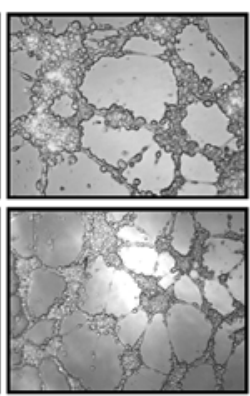

VEGF/siDAPK

Figure 1. DAPK promotes anti-angiogenic activity in HUVECs. (A) The inhibitory effect of DAPK on HUVEC proliferation. Cells were incubated with $10 \mathrm{ng} / \mathrm{ml}$ stimulator VEGF and then transfected with the control (expression vector only), DAPK, or DAPK-siRNA, respectively. The cpm value of [ $\left.{ }^{3} \mathrm{H}\right]$ thymidine was calculated using a liquid scintillation counter. Data are shown as the means \pm SD of three separate experiments. (B) Invasion of HUVECs was evaluated using the Transwell Boyden chamber. In the absence or presence of VEGF, HUVECs $\left(4.5 \times 10^{4}\right.$ cells) were transfected with the control (expression vector only), DAPK, or DAPK-siRNA, respectively. After $48 \mathrm{~h}$ incubation at $37^{\circ} \mathrm{C}$, invading cells were counted under a microscope and the mean values were estimated. The data are expressed as the means \pm SD of four separate experiments. (C) Inhibitory effect of DAPK on HUVEC tube formation. HUVECs $\left(9.5 \times 10^{4}\right)$ were cultured in 24-well plates containing growth factor reduced Matrigel, and pre-treated with/without VEGF, followed by transfection with the control, DAPK, or DAPKsiRNA. Capillary-like tubular structures were photographed with a digital camera attached to an inverted microscope. The tube lengths were estimated and the data are presented as the means $\pm \mathrm{SD}$. Three independent experiments were performed in triplicate. ${ }^{*} \mathrm{P}<0.05$ compared to the control. DAPK, death-associated protein kinase; HUVECs, human umbilical vein endothelial cells; VEGF, vascular endothelial growth factor.

A

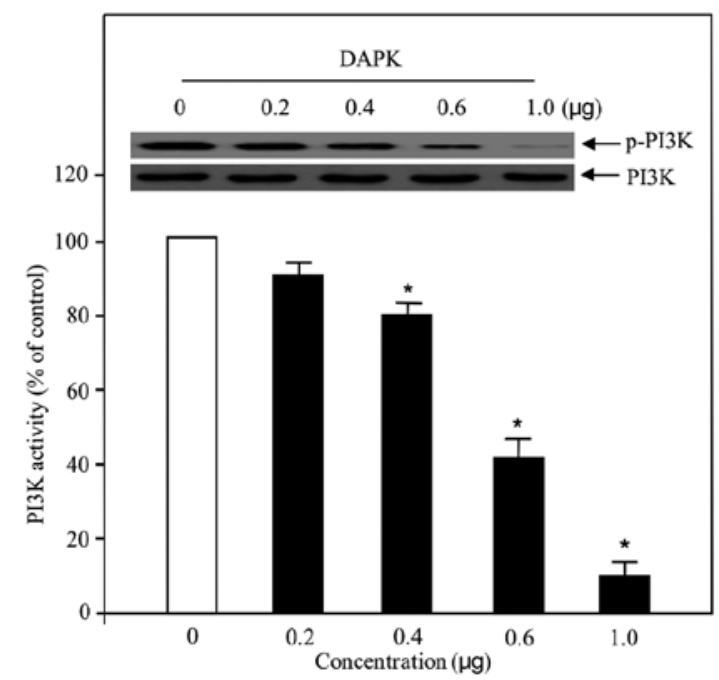

B

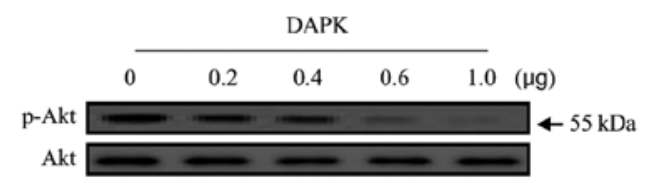

C



Figure 2. Effect of DAPK on PI3K activity and PI3K/Akt phosphorylation in carcinoma cells. (A) The inhibitory effect on PI3K activity, in the presence of DAPK, was evaluated using the in vitro PI3 kinase assay system (bottom panel). Experiments were performed in triplicate and error bars are shown as means \pm SD. ${ }^{*} \mathrm{P}<0.05$. PI3K was transfected with increasing concentrations of DAPK, collected and introduced to western blotting for the indicated dose proteins (upper panel). PI3K was used to verify equal loading of the samples. (B) Ovarian carcinoma cells were transfected with various concentrations of DAPK. The phosphorylation of Akt was determined using western blot analysis. (C) Cells were transfected with control (expression vector only), DAPK, rapamycin as an mTOR inhibitor, rapamycin plus DAPK and wortmannin as a PI3K inhibitor, respectively. After $24 \mathrm{~h}$, the cells were collected, lysed on ice-cold RIPA buffer and western blotting was carried out. Western blotting for unphosphorylated Akt and 4E-BP1 was used as a loading control. DAPK, death-associated protein kinase. 
A

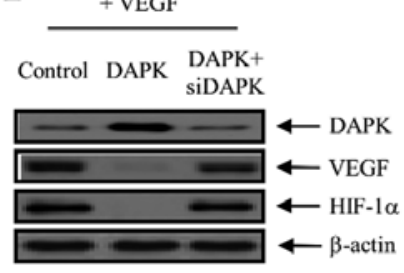

B



C

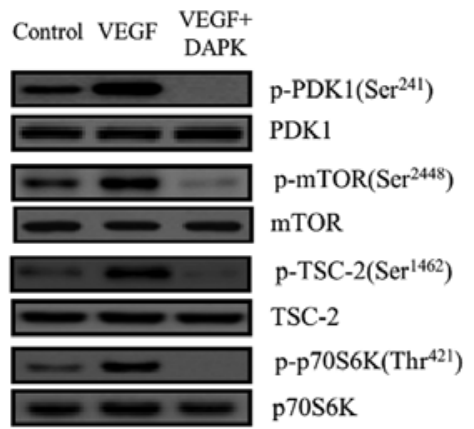

Figure 3. DAPK suppresses the expression of VEGF and HIF-1 $\alpha$ in carcinoma cells. (A) Cells were incubated with $10 \mathrm{ng} / \mathrm{ml}$ VEGF and then control (expression vector only)-transfected or transfected with DAPK or DAPK plus DAPK-siRNA. VEGF and HIF-1 $\alpha$ expression was then detected by immunoblotting. Three independent experiments were performed in triplicate. (B) DAPK inhibits hypoxia-induced VEGF and HIF-1 $\alpha$ activation. Cells were transfected with DAPK for $16 \mathrm{~h}$ under either normoxic or hypoxic conditions. Equal protein amounts were introduced to SDS-PAGE, blotted, and incubated with either specific primary VEGF, HIF-1 $\alpha$, or $\beta$-actin antibody. (C) After transfection with DAPK, cells were collected for western blot analysis with primary antibodies specific for the phosphorylated and non-phosphorylated proteins, which is indicative of PDK1 phosphorylation, mTOR phosphorylation, TSC-2 phosphorylation and the phosphorylation of downstream modulators, including p70S6K. The results shown are representative of three independent experiments. DAPK, death-associated protein kinase; VEGF, vascular endothelial growth factor; HIF-1 $\alpha$, hypoxia-inducible factor-1 $\alpha$; PDK1, phosphoinositide-dependent protein kinase-1; TSC-2, tuberous sclerosis complex-2.

DAPK inhibits the expression of VEGF and HIF-1 $\alpha$ in ovarian cancer cells. In rapidly growing cancer, hypoxic conditions strongly activate the expression of the transcription factor HIF-1 $\alpha$, which in turn stimulates the expression of VEGF proteins in carcinoma cells. VEGF expression levels modulate the effects of other angiogenic regulators and therefore play key roles in the regulation of tumor angiogenesis. In order to disrupt new blood vessel formation in cancer, it is key to suppress the expression of the VEGF and HIF-1 $\alpha$ proteins in carcinoma cells. To address whether DAPK inhibits the level of VEGF and HIF-1 $\alpha$ protein expression via the PI3K/Akt signaling pathway, we first evaluated the inhibitory effect of DAPK on VEGF expression in SKOV-3 ovarian carcinoma cells. As presented in Fig. 3A, overexpression of DAPK clearly decreased the VEGF expression level, whereas DAPK-siRNA had no effect. We also measured the effect of DAPK on HIF-1 $\alpha$ protein expression. HIF- $1 \alpha$ is a key regulator for VEGF expression as a transcription factor. As indicated in Fig. 3A, DAPK markedly decreased the expression of the HIF-1 $\alpha$ protein. On the other hand, the inhibitory effect of DAPK was completely restored by DAPK-siRNA transfection. Subsequently, we measured the levels of HIF-1 $\alpha$ and VEGF protein expression in SKOV-3 cells exposed to normoxia or $1 \% \mathrm{O}_{2}$ hypoxia and DAPK transfection. After a 16-h treatment, levels of HIF-1 $\alpha$ and VEGF protein expression indicated that they were fully activated. In contrast, HIF-1 $\alpha$ and VEGF expression levels were rapidly reduced after DAPK transfection (Fig. 3B). Therefore, it may be suggested that hypoxia significantly promoted the levels of HIF-1 $\alpha$ and VEGF expression, whereas DAPK suppressed their activation. Next, we examined the effects of DAPK on the downstream regulators in the PI3K/ Akt signaling pathway. DAPK significantly suppressed the phosphorylation of mTOR on the $\mathrm{Ser}^{2448}$ residue position, tuberous sclerosis complex-2 (TSC-2) on the $\mathrm{Ser}^{1462}$ residue position, and p70 ribosomal protein S6 kinase (p70S6K) on the $\mathrm{Thr}^{421}$ residue position, which are downstream factors essential to mTOR (Fig. 3C). These results indicate that DAPK inhibits the autocrine effect of VEGF in endothelial cells and, therefore, has a direct anti-angiogenic effect, which leads to the inhibition of tumor angiogenesis, metastasis and tumorigenesis.

\section{DAPK inhibits VEGF-induced VEGFR-2 phosphorylation} through the interaction with VEGFR-2 protein. VEGFR-2 is a key modulator of the VEGF-induced endothelial cell involved cellular based-physiological/pathological function. To explore the biological/functional relevance of the association between VEGFR-2 and DAPK, we evaluated the effect of DAPK on VEGF-stimulated VEGFR-2 phosphorylation in HUVECs. As shown in Fig. 4A, overexpression of DAPK markedly decreased VEGF-stimulated VEGFR-2 phosphorylation, while DAPKsiRNA had no effect. These new findings indicated that DAPK strongly suppressed protein levels of VEGFR-2 phosphorylation in vitro in HUVECs. Direct interaction between the two proteins is crucial for the majority of cellular biological/physiological mechanisms. For example, signal transductions from the exterior of the cell are specifically mediated to the inside of the cell by protein-protein interactions of the signaling components. This process plays a crucial role in the cellular biological mechanisms and in various diseases involving aggressive solid cancer. Protein-protein interactions are central to almost 
A

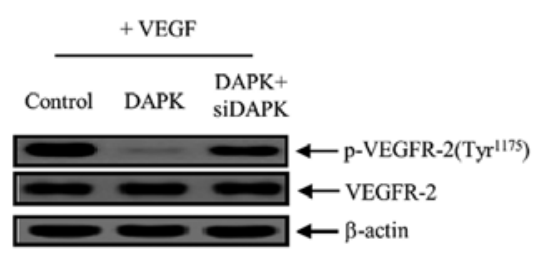

C

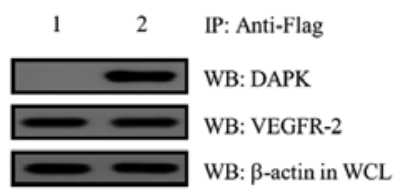

B vector only VEGFR-1 VEGFR-2

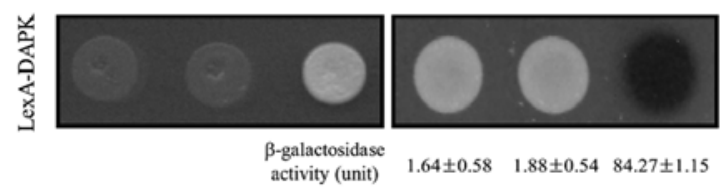

D
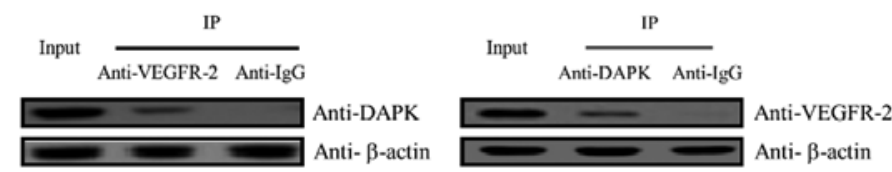

$\mathbf{E}$



Figure 4. DAPK inhibits VEGF-induced VEGFR-2 phosphorylation through the interaction with VEGFR-2 protein. (A) HUVECs were treated with VEGF angiogenesis inducer and then transfected with the control (expression vector only), DAPK, or DAPK plus DAPK-siRNA, respectively. Phosphorylation of VEGFR-2 was detected using the indicated specific primary antibody. Unphosphorylated VEGFR-2 and $\beta$-actin were used as the loading control. (B) Physical interaction between GABARBP and VEGFR-2. Direct interactions were observed by monitoring cell growth on deplete-leucine amino acid plate, and through the formation of a black colony on the cultured plate containing the X-gal reagent $(37,38)$. The values of the $\beta$-galactosidase activity (unit), calculated by adding $o$-nitrophenyl $\beta_{-\mathrm{D}}$-galactopyranoside (ONPG) assays, are indicated below the corresponding lanes $(37,38)$. (C) Co-immunoprecipitation of DAPK with VEGFR-2. Immunoprecipitation began by using primary anti-Flag antibody with lysates from both transfected HEK293T cells. After immunoprecipitation, precipitated proteins were visualized using the primary anti-DAPK and anti-VEGFR-2 antibody. Lane 1, pcDNA3.1 (expression vector only) and pcDNA3.1/Flag-DAPK transfectant; lane 2, pcDNA3.1/Flag-DAPK and pcDNA3.1-VEGFR-2 transfectant. (D) In vitro co-immunoprecipitation between the endogenous VEGFR-2 and DAPK shows the interactions of the two proteins. (E) Inhibition of the VEGFR-2-dependent transcriptional activity by DAPK. HUVECs were co-transfected with $500 \mathrm{ng}$ of VEGFR-2-Luc, $500 \mathrm{ng}$ of a VEGFR-2 inserted expression vector (pcDNA3.1/VEGFR-2) and increasing concentrations of gene-encoding DAPK (pcDNA3.1/Flag-DAPK) (100, 250 and $500 \mathrm{ng}$ ). The results shown are representative of at least three independent experiments. The values are expressed as the means $\pm \mathrm{SD}$. ${ }^{*} \mathrm{P}<0.05$ compared to the control. DAPK, death-associated protein kinase; VEGF, vascular endothelial growth factor; HUVECs, human umbilical vein endothelial cells; VEGFR-2, VEGF receptor-2.

every process in the living cell. Thus, we also demonstrated the interaction between DAPK and VEGFR-2 protein in an in vivo and in vitro system. VEGFR-1 and expression vector only were used as the negative control. As shown in Fig. 4B, DAPK with VEGFR-2, but not with VEGFR-1 and expression vector only, permitted yeast cell growth on a leucine-depleted plate, and $\beta$-galactosidase activity between DAPK and VEGFR-2 was fully activated (84.27 \pm 1.15$)$. However, control (vector only) (1.64 \pm 0.58$)$ and VEGFR-1 (1.88 \pm 0.54$)$ expression activity failed, suggesting a specific interaction between DAPK and VEGFR-2. Consistent with these results, direct interactions between DAPK and VEGFR-2 were confirmed using co-immunoprecipitation in vitro assays. Recombinant plasmids of DAPK (pcDNA3.1/Flag-DAPK) and VEGFR-2 (pcDNA3.1-VEGFR-2), or pcDNA3.1/Flag-DAPK and expres- sion vector only (pcDNA3.1) were co-transfected into the HEK293T cells. Subsequently, an immunoprecipitation was incubated using anti-Flag specific primary antibody with total cell lysates from both transfected cells. After immunoprecipitation, the precipitated proteins were detected with a specific anti-DAPK or anti-VEGFR-2 primary antibody. As indicated in Fig. 4C, pcDNA3.1-VEGFR-2 was co-immunoprecipitated with pcDNA3.1/Flag-DAPK (lane 2 in upper panel), whereas it failed with pcDNA3.1 plasmid (expression vector only) (lane 1 in upper panel). At the same time, in order to confirm the direct interaction between endogenous DAPK and the VEGFR-2 protein in cellular physiological conditions, we subjected this construct to immunoprecipitation with the proteins endogenously expressed in the HEK293T cells using a specific anti-DAPK or anti-VEGFR-2 primary antibody. The endog- 
enous DAPK protein was then directly co-immunoprecipitated with the VEGFR-2 protein (Fig. 4D). Our results strongly indicate the binding of endogenous VEGFR-2 to the DAPK protein in the cells. Next, to address whether DAPK regulates VEGFR-2, the effect of DAPK on VEGFR-2 transcription activity was also calculated by a luciferase reporter assay, using a construct integrating a VEGFR-2 promoter fused to the luciferase gene. The luciferase activity was gradually inhibited by transient transfection of DAPK in a concentration-dependent manner (Fig. 4E), providing further evidence of the importance of DAPK for the regulation of VEGFR-2 activity. These results strongly suggest that the overexpression of DAPK downregulates its transcriptional activity. The results validated that DAPK obstructs the VEGFR-2 transcriptional activation level by inhibiting VEGFR-2 phosphorylation via the PI3K/Akt signaling cascade.

\section{Discussion}

In the present study, we demonstrated that the possible intracellular mechanisms of DAPK have an inhibitory effect on the roles of VEGF-induced angiogenesis in the endothelial cell system. In angiogenesis and vasculogenesis, VEGF is a key regulator serving as a potent cytokine in endothelial cell proliferation, migration and survival (37). Generally, angiogenic signaling cascades are a route mediated by VEGF and their receptors (VEGFRs) (38). VEGF expression is closely correlated with the regulation of tumor growth, metastasis and aggression, as well as poor survival $(27,39,40)$. Notably, most human solid tumors greatly increase VEGF and HIF-1 $\alpha$ expression, which promote tumor angiogenesis and tumor growth. Thus, inhibition of tumor angiogenesis by the interruption of VEGF and HIF-1 $\alpha$ expression is becoming a crucial approach for cancer treatment (41). One of many studies reported that the maximal suppression of tumor angiogenesis and growth can be accomplished by completely disturbing the circulation of VEGF (42). Herein, we established a new cellular molecular mechanism for DAPK as a novel potent angiogenic factor that may target through the disturbance of VEGF and HIF-1 $\alpha$ expression in the PI3K/Akt signaling cascade using a tumor model system. As presented in Fig. 1, ectopically expressed DAPK markedly inhibited major events in VEGFinduced angiogenesis in vitro, which involved endothelial cell proliferation and cell migration. Meanwhile, overexpression of DAPK completely abrogated the VEGF-induced capillary-like tubular structure network. In the presence of DAPK-siRNA, the endothelial cells were nearly none affected. Collectively, these results strongly indicate that DAPK specifically mediates VEGF-induced HUVEC migration and tube formation.

HIF-1 increases the transcriptional levels of various genes, including VEGF in tumor progression (43). HIF-1 $\alpha$ is frequently overexpressed in several human solid cancers (44), and its activation is closely associated with tumor angiogenesis and tumorigenesis progression in cells (45). Additionally, HIF-1 upregulates the transcription of the VEGF gene by binding to the hypoxia-response element (HRE) in the VEGF promoter region (46). Fang et al (47) reported that apigenin significantly suppresses the expression of HIF-1 $\alpha$ and VEGF in ovarian carcinoma cells. As mentioned above, HIF-1 $\alpha$ is a major modulator for VEGF expression as a transcription factor. As shown in Fig. 3A, DAPK strongly downregulated the expression of HIF-1 $\alpha$ protein. In contrast, the inhibitory effect of DAPK was completely restored by DAPK-siRNA transfection. Subsequently, we assessed the levels of HIF-1 $\alpha$ and VEGF protein expression in SKOV-3 ovarian carcinoma cells exposed to normoxia or $1 \% \mathrm{O}_{2}$ hypoxia and DAPK transfection. After a 16-h treatment, the levels of HIF-1 $\alpha$ and VEGF protein expression indicated that they were fully activated. On the other hand, levels of HIF-1 $\alpha$ and VEGF protein expression rapidly decreased after DAPK transfection (Fig. 3B). Collectively, conditions of hypoxia were significantly upregulated in the levels of HIF-1 $\alpha$ and VEGF protein expression, whereas DAPK inhibited its activation.

VEGF phosphorylates through the interaction with VEGFR-2 and its downstream signaling component fully activates PI3K/Akt phosphorylation, which is a potent cytokine in the cellular control of endothelial cell growth and survival of various types of tumor, including ovarian cancer (48-51). Akt activates mTOR and is mediated by mTOR via a negative- and positive-feedback biological system (52), while also acting as a key regulator for cell proliferation, enhancing cell survival through various biological mechanisms. Furthermore, Akt is involved in that Akt1 and Akt3, two downstream modulators of the PI3K signaling pathway, have their crucial roles in ovarian tumorigenesis played via control of VEGF secretion and angiogenesis $(53,54)$. In spite of these reports, the functional mechanisms of ovarian tumor angiogenesis are still not understood. In the present study, we found that DAPK can inhibit the phosphorylation of VEGF-induced $\mathrm{PI} 3 \mathrm{~K}$ and Akt in a dose-dependent manner in vitro (Fig. 2). In contrast, DAPK-siRNA transfection fully restored DAPKreduced phosphorylation of both PI3K and Akt (data not shown). This inhibitory effect was comparable to that of other well-known PI3K inhibitors, such as wortmannin and rapamycin, which are well-known mTOR signaling pathways. As presented in Fig. 2C, DAPK clearly downregulated the phosphorylation of Akt (Ser473) and the phosphorylation of 4E-BP1 (Tyr37/46), as well as the phosphorylation of PDK1 (Ser241) (Fig. 3C), one of the best characterized targets of the mTOR complex. Subsequently, ectopic expression of DAPK significantly reduced VEGF-induced VEGFR-2 phosphorylation, whereas DAPK-siRNA did not have any effect. This new finding suggests that DAPK strongly inhibits the levels of VEGFR-2 phosphorylation in vitro in HUVECs. The direct interaction between two proteins is important for the majority of the cellular biological/functional mechanisms (Fig. 4). Collectively, the results indicate that DAPK can obstruct VEGFR-2 transcriptional activity by inhibiting VEGFR-2 phosphorylation through the PI3K/Akt signaling cascade.

In summary, we propose that the phosphorylation of VEGFR-2 has an influence on the cellular biological function of tumor angiogenesis. In the present study, we further validated a new biological/physiological function and cellular molecular mechanism for DAPK accurately, which serves as a novel potential anti-angiogenic factor that can mediate the phosphorylation of $\mathrm{PI} 3 \mathrm{~K} / \mathrm{mTOR} / 4 \mathrm{E}-\mathrm{BP} 1$ via the interruption of the VEGF and HIF-1 $\alpha$ expression levels. Therefore, a combination therapy with DAPK and traditional drugs may be a useful approach for more advanced ovarian tumor, recurrent and certain malignant patients. 


\section{Acknowledgements}

This study was supported by grants from the National Cancer Center (NCC-1210470-2).

\section{References}

1. Cohen O, Inbal B, Kissil JL, et al: DAP-kinase participates in TNF- $\alpha-$ and Fas-induced apoptosis and its function requires the death domain. J Cell Biol 146: 141-148, 1999.

2. Jang $\mathrm{CW}$, Chen $\mathrm{CH}$, Chen $\mathrm{CC}$, et al: TGF- $\beta$ induces apoptosis through Smad-mediated expression of DAP-kinase. Nat Cell Biol 4: 51-58, 2002.

3. Bialik S and Kimchi A: The death-associated protein kinases: structure, function, and beyond. Annu Rev Biochem 75: 189-210, 2006.

4. Deiss LP, Feinstein E, Berissi H, Cohen O and Kimchi A: Identification of a novel serine/threonine kinase and a novel $15-\mathrm{kD}$ protein as potential mediators of the gamma interferon-induced cell death. Genes Dev 9: 15-30, 1995.

5. Cohen O,Feinstein E and Kimchi A: DAP-kinase is $\mathrm{Ca}^{2+} /$ calmodulin-dependent, cytoskeletal-associated protein kinase, with cell death-inducing functions that depend on its catalytic activity. EMBO J 16: 998-1008, 1997.

6. Raveh T, Droguett G, Horwitz MS, DePinho RA and Kimchi A DAP kinase activates a p19ARF/p53-mediated apoptotic checkpoint to suppress oncogenic transformation. Nat Cell Biol 3 : $1-7,2001$

7. Michie AM, McCaig AM, Nakagawa R and Vukovic M: Deathassociated protein kinase (DAPK) and signal transduction: regulation in cancer. FEBS J 277: 74-80, 2010.

8. Sanchez-Cespedes M, Esteller M, Wu L, et al: Gene promoter hypermethylation in tumors and serum of head and neck cancer patients. Cancer Res 60: 892-895, 2000.

9. Kim DH, Nelson HH, Wiencke JK, et al: Promoter methylation of DAP-kinase: association with advanced stage in non-small cell lung cancer. Oncogene 20: 1765-1770, 2001.

10. Dansranjavin T, Möbius C, Tannapfel A, et al: E-cadherin and DAP kinase in pancreatic adenocarcinoma and corresponding lymph node metastases. Oncol Rep 15: 1125-1131, 2006.

11. Kissil JL, Feinstein E, Cohen O, et al: DAP-kinase loss of expression in various carcinoma and B-cell lymphoma cell lines: possible implications for role as tumor suppressor gene. Oncogene 15: 403-407, 1997.

12. Katzenellenbogen RA, Baylin SB and Herman JG: Hypermethylation of the DAP-kinase $\mathrm{CpG}$ island is a common alteration in B-cell malignancies. Blood 93: 4347-4353, 1999.

13. Henshall DC, Araki T, Schindler CK, et al: Expression of deathassociated protein kinase and recruitment to the tumor necrosis factor signaling pathway following brief seizures. J Neurochem 86: $1260-1270,2003$.

14. Anjum R, Roux PP, Ballif BA, Gygi SP and Blenis J: The tumor suppressor DAP kinase is a target of RSK-mediated survival signaling. Curr Biol 15: 1762-1767, 2005.

15. Chen CH, Wang WJ, Kuo JC, et al: Bidirectional signals transduced by DAPK-ERK interaction promote the apoptotic effect of DAPK. EMBO J 24: 294-304, 2005.

16. Liambi F, Lourenço FC, Gozuacik D, et al: The dependence receptor UNC5H2 mediates apoptosis through DAP-kinase. EMBO J 24: 1192-1201, 2005.

17. Tang X, Khuri FR, Lee JJ, et al: Hypermethylation of the deathassociated protein (DAP) kinase promoter and aggressiveness in stage I non-small-cell lung cancer. J Natl Cancer Inst 92: 1511-1516, 2000.

18. Harden SV, Tokumaru Y, Westra WH, et al: Gene promoter hypermethylation in tumors and lymph nodes of stage I lung cancer patients. Clin Cancer Res 9: 1370-1375, 2003.

19. Lu C, Soria JC, Tang X, et al: Prognostic factors in resected stage I non-small-cell lung cancer: A multivariate analysis of six molecular markers. J Clin Oncol 22: 4575-4583, 2004.

20. Risau W: Mechanisms of angiogenesis. Nature 386: 671-674, 1997.

21. Denny WA: Prodrug strategies in cancer therapy. Eur J Med Chem 36: 577-595, 2001.

22. Blagosklonny MV: Antiangiogenic therapy and tumor progression. Cancer Cell 5: 13-17, 2004.
23. Olson TA, Mohanraj D, Carson LF and Ramakrishnan S: Vascular permeability factor gene expression in normal and neoplastic human ovaries. Cancer Res 54: 276-280, 1994.

24. Paley PJ, Staskus KA, Gebhard K, et al: Vascular endothelial growth factor expression in early stage ovarian carcinoma. Cancer 80: 98-106, 1997.

25. Ohta Y, Tomita Y, Oda M, et al: Tumor angiogenesis and recurrence in stage I non-small cell lung cancer. Ann Thorac Surg 68: 1034-1038, 1999

26. Ishigami SI, Arii S, Furutani M, et al: Predictive value of vascular endothelial growth factor (VEGF) in metastasis and prognosis of human colorectal cancer. Br J Cancer 78: 1379-1384, 1998.

27. Yamamoto S, Konishi I, Mandai M, et al: Expression of vascular endothelial growth factor (VEGF) in epithelial ovarian neoplasms: correlation with clinicopathology and patient survival, and analysis of serum VEGF levels. Br J Cancer 76: 1221-1227, 1997.

28. Spannuth WA, Nick AM, Jennings NB, et al: Functional significance of VEGFR-2 on ovarian cancer cells. Int J Cancer 124: 1045-1053, 2009.

29. Lee OH, Kim YM, Lee YM, et al: Sphingosine 1-phosphate induces angiogenesis: its angiogenic action and signaling mechanism in human umbilical vein endothelial cells. Biochem Biophys Res Commun 264: 743-750, 1999.

30. Byun HJ, Lee JH, Kim BR, et al: Anti-angiogenic effects of thioridazine involving the FAK-mTOR pathway. Microvasc Res 84: 227-234, 2012.

31. Senger DR, Ledbetter SR, Claffey KP, et al: Stimulation of endothelial cell migration by vascular permeability factor/vascular endothelial growth factor through cooperative mechanisms involving the alphavbeta3 integrin, osteopontin, and thrombin. Am J Pathol 149: 293-305, 1996.

32. Benelli R and Albini A: In vitro models of angiogenesis: the use of Matrigel. Int J Biol Markers 14: 243-246, 1999.

33. Fruman DA, Mauvais-Jarvis F, Pollard DA, et al: Hypoglycaemia, liver necrosis and perinatal death in mice lacking all isoforms of phosphoinositide 3-kinase p85 $\alpha$. Nat Genet 26: 379-382, 2000.

34. Lee KB, Byun HJ, Park SH, et al: CYR61 controls p53 and NF- $\kappa B$ expression through PI3K/Akt/mTOR pathways in carboplatininduced ovarian cancer cells. Cancer Lett 315: 86-95, 2012.

35. Rho SB, Kim MJ, Lee JS, et al: Genetic dissection of proteinprotein interactions in multi-tRNA synthetase complex. Proc Natl Acad Sci USA 96: 4488-4493, 1999.

36. Rho SB, Song YJ, Lim MC, et al: Programmed cell death 6 (PDCD6) inhibits angiogenesis through $\mathrm{PI} 3 \mathrm{~K} / \mathrm{mTOR} / \mathrm{p} 70 \mathrm{~S} 6 \mathrm{~K}$ pathway by interacting of VEGFR-2. Cell Signal 24: 131-139, 2012.

37. Plate KH: Control of tumor growth via inhibition of tumor angiogenesis. Adv Exp Med Biol 451: 57-61, 1998.

38. Ferrara N: Role of vascular endothelial growth factor in physiologic and pathologic angiogenesis: therapeutic implications. Semin Oncol 29 (Suppl 16): S10-S14, 2002.

39. Mu J, Abe Y, Tsutsui T, et al: Inhibition of growth and metastasis of ovarian carcinoma by administering a drug capable of interfering with vascular endothelial growth factor activity. Jpn J Cancer Res 87: 963-971, 1996.

40. Hartenbach EM, Olson TA, Goswitz JJ, et al: Vascular endothelial growth factor (VEGF) expression and survival in human epithelial ovarian carcinomas. Cancer Lett 121: 169-175, 1997.

41. Ferrara N and Davis-Smyth T: The biology of vascular endothelial growth factor. Endocr Rev 18: 4-25, 1997.

42. Gerber HP, Kowalski J, Sherman D, Eberhard DA and Ferrara N: Complete inhibition of rhabdomyosarcoma xenograft growth and neovascularisation requires blockade of both tumor and host vascular endothelial growth factor. Cancer Res 60: 6253-6258, 2000.

43. Semenza GL: Hypoxia, clonal selection, and the role of HIF-1 in tumor progression. Crit Rev Biochem Mol Biol 35: 71-103, 2000.

44. Zhong H, De Marzo AM, Laughner E, et al: Overexpression of hypoxia-inducible factor $1 \alpha$ in common human cancers and their metastases. Cancer Res 59: 5830-5835, 1999.

45. Maxwell PH, Dachs GU, Gleadle JM, et al: Hypoxia-inducible factor-1 modulates gene expression in solid tumors and influences both angiogenesis and tumor growth. Proc Natl Acad Sci USA 94: 8104-8109, 1997.

46. Forsythe JA, Jiang BH, Iyer NV, et al: Activation of vascular endothelial growth factor gene transcription by hypoxia-inducible factor 1. Mol Cell Biol 16: 4604-4613, 1996. 
47. Fang J, Xia C, Cao Z, et al: Apigenin inhibits VEGF and HIF-1 expression via PI3K/AKT/p70S6K1 and HDM2/p53 pathways. FASEB J 19: 342-353, 2005.

48. Altomare DA, Wang HQ, Skele KL, et al: AKT and mTOR phosphorylation is frequently detected in ovarian cancer and can be targeted to disrupt ovarian tumor cell growth. Oncogene 23: 5853-5857, 2004.

49. Olsson AK, Dimberg A, Kreuger J and Caesson-Welsh L: VEGF receptor signaling - in control of vascular function. Nat Rev Mol Cell Biol 7: 359-371, 2006.

50. Engelman JA: Targeting PI3K signalling in cancer: opportunities, challenges and limitations. Nat Rev Cancer 9: 550-562, 2009.
51. Hanrahan AJ, Schultz N, Westfal ML, et al: Genomic complexity and AKT dependence in serous ovarian cancer. Cancer Discov 2: 56-67, 2012.

52. Guertin DA and Sabatini DM: An expanding role for mTOR in cancer. Trends Mol Med 11: 353-361, 2005.

53. Xia C, Meng Q, Cao Z, Shi X and Jiang BH: Regulation of angiogenesis and tumor growth by 110 alpha and AKT1 via VEGF expression. J Cell Physiol 209: 56-66, 2006.

54. Liby TA, Spyropoulos P, Buff Lindner H, et al: Akt3 controls vascular endothelial growth factor secretion and angiogenesis in ovarian cancer cells. Int J Cancer 130: 532-543, 2012. 